\title{
Light-emitting diode-based transcranial photoacoustic measurement of sagittal sinus oxyhemoglobin saturation in hypoxic neonatal piglets
}

\author{
Jeeun Kang, ${ }^{1,2}$ Raymond C. Koehler, ${ }^{3}$ Shawn Adams, ${ }^{3}$ Ernest M. Graham, ${ }^{4,5}$ Emad M. \\ Boctor $^{1,2 *}$ \\ ${ }^{1}$ Department of Radiology and Radiological Science, Johns Hopkins University School of Medicine \\ (JHU SOM), Baltimore, MD 21205, USA; \\ ${ }^{2}$ Laboratory for Computational Sensing and Robotics, Johns Hopkins University Whiting School of \\ Engineering, Baltimore, MD 21218, USA \\ ${ }^{3}$ Department of Anesthesiology and Critical Care Medicine, Johns Hopkins University School of \\ Medicine, Baltimore, MD 21205, USA \\ ${ }^{4}$ Division of Maternal-Fetal Medicine, Department of Gynecology-Obstetrics, Johns Hopkins \\ University School of Medicine, Baltimore, MD 21205, USA; \\ ${ }^{5}$ Neuroscience Intensive Care Nursery Program, Johns Hopkins University School of Medicine, \\ Baltimore, Maryland, MD 21205, USA
}

*Corresponding author:

Emad M. Boctor

eboctor1@jhmi.edu

\begin{abstract}
We present a light-emitting diode (LED)-based transcranial photoacoustic measurement (LED-trPA) of oxyhemoglobin $\left(\mathrm{HbO}_{2}\right)$ saturation at superior sagittal sinus (SSS) in hypoxic neonatal piglets. The optimal LED imaging wavelengths and frame averaging scheme were determined based on in vivo characterization of transcranial sensitivity. Based on the framework (690/850 $\mathrm{nm}$ with $>20$ frame averaging), graded hypoxia was successfully identified in neonatal piglets in vivo with less than $10.0 \%$ of root mean squared error (RMSE). This preclinical study suggests the feasibility of a rapid, cost-effective, and safe LED-trPA monitoring of perinatal hypoxia-ischemia and prompt interventions for clinical use.
\end{abstract}

\section{Main text}

Photoacoustic (PA) imaging is an emerging hybrid modality providing molecular contrast of light absorbance in biological tissue at submillimeter-scale spatial resolution and centimeter-scale penetration depth [1]. Several transcranial PA sensing methods have been presented to quantify electrophysiologic and neurophysiologic dynamics mostly in rodent brain [2-6]. We recently validated the feasibility of transcranial PA measurement of graded oxyhemoglobin $\left(\mathrm{HbO}_{2}\right)$ saturation changes in the superior sagittal sinus (SSS) in a neonatal piglet model simulating a skull thickness and brain development comparable to those of term human neonates [7]. This validation suggests its high translationability for monitoring the incidence of perinatal hypoxic-ischemic encephalopathy (HIE). However, this methodology still conveys concerns remaining on the use of a class-IV high-energy Nd:YAG laser equipped with a tunable optical parametric oscillator (OPO), regarding laser safety, slow scanning speed, bulkiness, and high cost.

There have been several investigations to make PA imaging system safer, faster, more compact, and affordable. A compact approach for brain sensing has been presented using pulsed laser diode (PLD) technology with up to 1-4 mJ per pulse with $\sim 100$-nsec pulse duration and $1 \mathrm{kHz}$ pulse-repetition frequency (PRF) $[8,9]$. However, the study only demonstrated a monochrome or single-point reading, lacking spectral and spatial specificity, 
respectively. On the other hand, light-emitting diode (LED)-based PA imaging has been receiving attention at the forefront of translational investigations [10]. State-of-the-art LED technology provides discrete near-infrared wavelengths $(690,750,820,850,940$, and $980 \mathrm{~nm})$ at up to $200 \mu \mathrm{J}$ per pulse with $30-100$-nsec pulse duration and $0.2-16 \mathrm{KHz}$ PRF. Notably, the LED source offers no need for safety goggles and barriers which are strictly obliged when using high-energy lasers. Furthermore, its unit cost is below $10 \%$ from what is needed for an Nd:YAG OPO laser ( \$10-15K vs. \$70-200K). However, to the best of our knowledge, transcranial sensing applications with LEDs have remained unexplored due to the challenges with high attenuation in the scalp and skull layers. In this short communication, we validate the feasibility of multi-spectral LED-based transcranial PA measurement (LED-trPA) of sagittal sinus $\mathrm{HbO}_{2}$ saturation in neonatal piglets with graded hypoxia in vivo. Our LED-trPA imaging system was built with 4-channel pulsed LED source (Prexion Inc., Japan) and ultrasound research package (Vantage 256, Verasonics Inc., USA) (Fig. 1).

All procedures on piglets were approved by the Johns Hopkins University Animal Care and Use Committee. Transcranial energy density threshold for SSS sensing $\left(\bar{\Phi}_{S S S}\right)$ was first characterized in anesthetized and mechanically ventilated 3-5-day-old neonatal piglets (1.5-2.1 kg; fraction of inspired $\left.\mathrm{O}_{2}\left(\mathrm{FiO}_{2}\right)=0.33 ; n=4\right)$. In this signal characterization stage, we employed the experimental setup based on an Nd:YAG OPO laser (Phocus Inline, Opotek Inc., USA) to investigate a wide range of incident energy density [7]. Catheters were inserted into the SSS to obtain cortical venous blood samples for blood gas measurement of the ground-truth $\mathrm{HbO}_{2}$ saturation at SSS. The pulsed laser was delivered by an optical fiber bundle attached to a 5.2-MHz linear array (L7-5, ATL Inc., USA). 10 $\%$ of the OPO output was intercepted for simultaneous recording. The piglet head was scanned in near-infrared LED wavelengths (i.e., 690, 750, 820, and $850 \mathrm{~nm}$ ) with graded flash-to-Q-switch delays in the Nd:YAG laser: 200 (optimal), 205, 210, $215 \mu \mathrm{sec}$. A flash-to-Q-switch delay longer than $200 \mu \mathrm{sec}$ lowers the energy density at the fiber output: $-16.13,-32.34$, and $-47.78 \%$ for 205,210 , and $215 \mu$ sec in average over entire wavelength range. The $1.5 \times 1.5-$ $\mathrm{mm}^{2}$ region-of-interest (ROI) was selected on SSS to quantify a regional PA spectrum (Fig. 2a). Fig. 2b shows the PA sensitivity at SSS as a function of incident energy density at each wavelength, fitted to linear regression curves (Mathworks Inc., USA). A constraint was applied to intercept the origin point (i.e., no PA intensity at $0 \mathrm{~mJ} / \mathrm{cm}^{2}$ ). Note that the background noise level (BG) was measured without laser excitation, and then subtracted from the measured PA intensity. In the examples shown in Fig. $2 \mathrm{~b}$ with no frame averaging, the linear regression slopes normalized to the BG were 8.06, 7.10, 5.76, and 7.59 (i.e., PA intensity at SSS $/ \mathrm{mJ} \cdot \mathrm{cm}^{-2} / \mathrm{BG}$ ) at 690, 750, 820, and $850 \mathrm{~nm}$, respectively. This implies that $690 \mathrm{~nm}$ and $850 \mathrm{~nm}$ yield the higher transcranial sensitivity at the SSS per an energy density than others. The BG was lowered with a factor of $\sqrt{N}$, reflecting reciprocal signal-to-noise ratio (SNR) improvement $[11,12]$. The cross point between the fitted curves to the BG was then identified at each frame averaging scheme $(1,2,5,10,20,50,100,200,500,1,000,2,000$ frames), specifying the energy density delivering 0-dB SNR.

Fig. 2c shows the $\bar{\Phi}_{S S S}$ estimated under different frame averaging schemes. The influential factor should be combinational among (1) total hemoglobin absorbance at SSS; (2) optical / acoustic attenuation in scalp and skull layers; and (3) noise level in ultrasound system. In general, the spectral trend of $\bar{\Phi}_{S S S}$ followed the inversion of the total hemoglobin absorbance at the ground-truth $\mathrm{HbO}_{2}$ saturation in the piglets, i.e., $52.33 \pm 5.95 \%$ (i.e., $0.89,1.06$, 1.23 , and 1.13 when $\times 1,000$ at $690,750,820$, and $850 \mathrm{~nm}$ [13]), implying that more hemoglobin absorbance lowers $\bar{\Phi}_{S S S}$. Also, the optical transmission through skull and scalp layers is another significant factor. The skull will have more absorptive attenuation as one gets close to $650 \mathrm{~nm}$ and $900 \mathrm{~nm}$, while holding a relatively stable level between $700 \mathrm{~nm}$ to $850 \mathrm{~nm}$. [14,15]. On the other hand, the dominant absorber in the scalp layer is melanin which gradually increase optical attenuation as one gets close to shorter wavelength range [16]. The inversely proportional relationship was identified between $\bar{\Phi}_{S S S}$ and the number of frames averaged, as expected.

From the $\bar{\Phi}_{S S S}$ characterization, we selected $690 \mathrm{~nm}$ and $850 \mathrm{~nm}$ for LED-trPA to secure low $\bar{\Phi}_{S S S}$ and spectral independency at the same time. When 2 LED arrays were used for each wavelength, the LED-trPA probe yielded the energy density at the depth of subject surface $\left(\Phi_{L E D}\right): 32.86 \pm 2.23$ and $89.03 \pm 5.28 \mu \mathrm{J} / \mathrm{cm}^{2}$ at $690 \mathrm{~nm}$ and $850 \mathrm{~nm}$ both with $105 \mathrm{nsec}$ pulse duration (red bars in Fig. 2c). Statistical significance of $\Phi_{L E D}(<0.05)$ was obtained from $\bar{\Phi}_{S S S}$ when applied 20- and 5-frame averaging at $690 \mathrm{~nm}$ and $850 \mathrm{~nm}$, respectively: $(690 \mathrm{~nm})$ 
$119.99 \pm 42.65, \quad 84.85 \pm 30.12, \quad 53.67 \pm 19.08, \quad 37.98 \pm 13.48, \quad 26.87 \pm 9.53 * \quad(p=0.0094), \quad 16.99 \pm 6.02, \quad 12.02 \pm 4.25$, $8.48 \pm 3.02,5.38 \pm 1.93,3.76 \pm 1.33$, and $2.63 \pm 0.93 \mu \mathrm{J} / \mathrm{cm}^{2}$; $(850 \mathrm{~nm}) 113.00 \pm 35.98,79.90 \pm 25.44,50.55 \pm 16.11^{*}$ $(p=0.001), 35.73 \pm 11.36,25.28 \pm 8.04,15.98 \pm 5.12,11.28 \pm 3.61,8.00 \pm 2.54,5.08 \pm 1.61,3.58 \pm 1.12$, and $2.53 \pm 0.82$ $\mu \mathrm{J} / \mathrm{cm}^{2}$ for $1,2,5,10,20,50,100,200,500,1,000$, and 2,000 frame averaging. From the $\Phi_{L E D}$ and $\bar{\Phi}_{S S S}$, an expected SNR at SSS (i.e., $\mathrm{SNR}_{\mathrm{est}}$ ) was calculated at each wavelength and frame averaging scheme, assuming that the PA intensity is proportional to the effective energy density:

$$
\mathrm{SNR}_{\mathrm{est}}=10 \log _{10}\left(\frac{\Phi_{L E D}}{\bar{\Phi}_{S S S}}\right) .
$$

The LED-trPA system configuration was then applied in our following in vivo validation study $\left(\mathrm{FiO}_{2}=0.33, n=3\right)$. Four LED bars were aligned to a linear array transducer to obtain a coronal cross-section of piglet head (Fig. 1). Fig. 3a shows the LED-trPA images at 690 and $850 \mathrm{~nm}$ with 1, 20, 200, 2,000 frame averaging schemes. The 1.5 $\times 1.5$ $\mathrm{mm}^{2} \mathrm{SSS}$ region was again selected for the quantification of SNR:

$$
S N R_{L E D}=10 \log _{10}\left(\frac{I_{L E D}-B G}{B G}\right),
$$

where $I_{L E D}$ is the LED light-induced PA intensity at SSS. Fig. $3 \mathrm{~b}$ and Table 2 presents the SNR $\mathrm{est}_{\text {and SNR }}$ aED together for each frame averaging scheme at $690 \mathrm{~nm}$ and $850 \mathrm{~nm}$, respectively. Overall, the LED-trPA measurements showed a well-matched trend to the corresponding estimations over different frame averaging schemes with $0.35 \pm 0.43 \mathrm{~dB}$ and $1.06 \pm 0.12 \mathrm{~dB}$ of differences (mean \pm standard deviation, SD) at $690 \mathrm{~nm}$ and $850 \mathrm{~nm}$, respectively.

We then induced graded hypoxia in the neonatal piglets by progressively changing $\mathrm{FiO}_{2}$ level: $1.00,0.80$, 0.60, 0.50, $0.400 .33,0.20,0.18,0.16,0.15$. At each $\mathrm{FiO}_{2}$ step, a 5-min stabilization period was allowed before the LED-trPA. 2,000 LED-trPA imaging frames were obtained each, followed by the collection of arterial and SSS blood samples before translating to the next $\mathrm{FiO}_{2}$ level. In some experiments, data were not obtained due to low MAP or inability to draw blood from the SSS catheter. From the data collected, linear regression curves were reconstructed between ground truths (blood gas measurements) and the LED-trPA-based $\mathrm{HbO}_{2}$ saturation estimations (Fig. 4a). With 2,000-frame averaging, the linear regression slope was 0.77 and y-intercept at $10.54 \%$ with the goodness of fit $\left(\mathrm{R}^{2}\right)$ at 0.80 . Root mean squared error (RMSE) to the ground truth (unity curve) was $6.22 \%$, and the Bland-Altman estimation analysis presented no physiologically meaningful bias, having $-1.38 \pm 12.15 \%$ (mean $\pm 1.96 \mathrm{SD}$ ). The deviation of the accuracy was repeatedly evaluated for $1,2,5,10,20,50,100,200,500,1,000$ frame averaging schemes with 20 permutations. Fig. $4 c$ shows the changes in slope, $y$-intercept, $\mathrm{R}^{2}$, and RMSE for the linear regression curves with different frame averaging schemes. In general, more frame averaging yielded the progressively improved $\mathrm{HbO}_{2}$ saturation estimation. Notably, single-frame LED-trPA imaging failed to track the change in $\mathrm{HbO}_{2}$ saturation at SSS with $0.38 \pm 0.18$ of slope, $31.34 \pm 8.14$ of y-intercept, $0.19 \pm 0.13$ of $\mathrm{R}^{2}$, and $15.61 \pm 2.10$ of RMSE. Otherwise, the 20-frame averaging scheme, enabled the first positive SNR at SSS for both wavelengths (Fig. 3b), derived the first estimation point limiting the RMSE below $10 \%$ in $\mathrm{HbO}_{2}$ saturation (i.e., $8.44 \pm 1.03$, mean \pm SD). However, the linear regression curve still presented insufficient accuracy to track $\mathrm{HbO}_{2}$ saturation changes at SSS: $0.67 \pm 0.06$ of slope, $12.49 \pm 2.21$ of $y$-intercept, $0.62 \pm 0.09$ of $\mathrm{R}^{2}$. This can be explained by still insufficient SNR at SSS (i.e., $0.87 \mathrm{~dB}$ ) with 690-nm LED. From 50- to 1,000-frame averaging scheme, the gradual increase of correlation accuracy to ground truth was found, agreed with Bland-Altman estimation analysis showing no physiologically meaningful bias observed at low or high $\mathrm{HbO}_{2}$ saturation levels.

In this study, we presented framework for a non-invasive, real-time, and safe LED-trPA of SSS in neonatal piglets in vivo as a significant translational step towards a rapid detection of perinatal HIE, enabling immediate interventions for tissue salvage. We first characterized $\bar{\Phi}_{S S S}$ the minimal energy density threshold for SSS sensing, $\bar{\Phi}_{S S S}$, followed by in vivo LED-trPA evaluation of graded hypoxia in the neonatal piglets. The use of the neonatal piglet model provides skull thickness and brain development comparable to those in term human neonates $[17,18]$. Moreover, the pig has skin more similar to humans than rodents [19]. 
Several aspects of this approach will need to be considered for improving this methodology: (1) Even though the LED-trPA framework could provide RMSE less than 10\%, the linear regression presented limited accuracy when compared to our previous results with Nd:YAG OPO laser, even with 2,000 frame averaging: 0.77 vs. 1.00 in slope; $10.54 \%$ vs. $1.49 \%$ in y-intercept; 0.80 vs. 0.87 of $\mathrm{R}^{2}$ [7]. It may be suggesting more frame averaging, but we concern the error in spectral decomposition given the much broader linewidth in the LEDs than that in the Nd:YAG OPO laser (20-35 nm vs. 2-3 nm). It could be also the source of error in our SNR estimation (Fig. 3). The appropriate correction in spectral decomposition will enhance the estimation accuracy. (2) The abundant temporal information in LED-trPA opens another opportunity for further enhancement. For instance, our group presented a deep neural network that improved the LED-based PA imaging quality by employing features in temporal sequences [20]. The solution may significantly improve the sensitivity of LED-trPA. However, a great caution will be needed to preserve the spectral features at individual wavelengths. (3) Embedding the LED-trPA on ultra-compact ultrasound systems will facilitate its clinical translation [21,22]. In this integrative phase, strict considerations on thermal and electrical interferences would be needed to be in a compact form factor.

Funding. NIH National Institute of Heart, Lung and Blood (NIH NIHLB) (R01HL139543); Congressionally Directed Medical Research Programs, U.S. Department of Defense (CDMRP, US DoD) (W81XWH-18-1-0188). Maryland Innovation Initiative, TEDCO.

Disclosures. The authors declare no conflicts of interest.

\section{References}

[1] L.V. Wang, S. Hu, Photoacoustic tomography: in vivo imaging from organelles to organs., Science. 335 (2012) 1458 1462. doi:10.1126/science.1216210.

[2] J. Yao, L. Wang, J.-M. Yang, K.I. Maslov, T.T.W. Wong, L. Li, C.-H. Huang, J. Zou, L.V. Wang, Highspeed label-free functional photoacoustic microscopy of mouse brain in action, Nature Methods. 12 (2015) 407 410. doi:10.1038/nmeth.3336.

[3] J. Lv, S. Li, J. Zhang, F. Duan, Z. Wu, R. Chen, M. Chen, S. Huang, H. Ma, L. Nie, In vivo photoacoustic imaging dynamically monitors the structural and functional changes of ischemic stroke at a very early stage., Theranostics. 10 (2020) 816-828. doi:10.7150/thno.38554.

[4] X. Wang, Y. Pang, G. Ku, X. Xie, G. Stoica, L.V. Wang, Noninvasive laser-induced photoacoustic tomography for structural and functional in vivo imaging of the brain, Nature Biotechnology. 21 (2003) 803 806. doi:10.1038/nbt839.

[5] J. Kang, S.D. Kadam, J.S. Elmore, B.J. Sullivan, H. Valentine, A.P. Malla, M.M. Harraz, A. Rahmim, J.U. Kang, L.M. Loew, M.H. Baumann, A.A. Grace, A. Gjedde, E.M. Boctor, D.F. Wong, Transcranial photoacoustic imaging of NMDA-evoked focal circuit dynamics in the rat hippocampus., J Neural Eng. 17 (2020) 025001. doi:10.1088/1741-2552/ab78ca.

[6] J. Kang, H.K. Zhang, S.D. Kadam, J. Fedorko, H. Valentine, A.P. Malla, P. Yan, M.M. Harraz, J.U. Kang, A. Rahmim, A. Gjedde, L.M. Loew, D.F. Wong, E.M. Boctor, Transcranial Recording of Electrophysiological Neural Activity in the Rodent Brain in vivo Using Functional Photoacoustic Imaging of Near-Infrared Voltage-Sensitive Dye, Front Neurosci-Switz. $13 \quad$ (2019) 579. doi:10.3389/fnins.2019.00579.

[7] J. Kang, E.M. Boctor, S. Adams, E. Kulikowicz, H.K. Zhang, R.C. Koehler, E.M. Graham, Validation of noninvasive photoacoustic measurements of sagittal sinus oxyhemoglobin saturation in hypoxic neonatal piglets, J Appl Physiol. 125 (2018) 983-989. doi:10.1152/japplphysiol.00184.2018.

[8] S.K. Kalva, P.K. Upputuri, M. Pramanik, High-speed, low-cost, pulsed-laser-diode-based secondgeneration desktop photoacoustic tomography system, Opt Lett. 44 (2018) 81. doi:10.1364/ol.44.000081. 
[9] R.O. Esenaliev, Optoacoustic diagnostic modality: From idea to clinical studies with highly compact laser diode-based systems, J Biomed Opt. 22 (2017) 091512. doi:10.1117/1.jbo.22.9.091512.

[10] Y. Zhu, T. Feng, Q. Cheng, X. Wang, S. Du, N. Sato, M.K.A. Singh, J. Yuan, Towards Clinical Translation of LED-Based Photoacoustic Imaging: A Review., Sensors Basel Switz. 20 (2020) 2484. doi:10.3390/s20092484.

[11] Y. Liang, J.-W. Liu, L. Wang, L. Jin, B.-O. Guan, Noise-reduced optical ultrasound sensor via signal duplication for photoacoustic microscopy, Opt Lett. 44 (2019) 2665. doi:10.1364/ol.44.002665.

[12] J. Kang, H.K. Zhang, A. Rahmim, D.F. Wong, J.U. Kang, E.M. Boctor, Toward high-speed transcranial photoacoustic imaging using compact near-infrared pulsed LED illumination system, in: 2017: p. 1176. doi: $10.1117 / 12.2253322$.

[13] S. Prahl, Optical Absorption of Hemoglobin, (1999).

[14] F. Salehpour, P. Cassano, N. Rouhi, M.R. Hamblin, L.D. Taboada, F. Farajdokht, J. Mahmoudi, Penetration Profiles of Visible and Near-Infrared Lasers and Light-Emitting Diode Light Through the Head Tissues in Animal and Human Species: A Review of Literature., Photobiomodulation Photomed Laser Surg. 37 (2019) 581-595. doi:10.1089/photob.2019.4676.

[15] M. Firbank, M. Hiraoka, M. Essenpreis, D.T. Delpy, Measurement of the optical properties of the skull in the wavelength range 650-950 nm, Phys Med Biol. 38 (1993) 503-510. doi:10.1088/0031-9155/38/4/002.

[16] S. Chinnathambi, N. Shirahata, Recent advances on fluorescent biomarkers of near-infrared quantum dots for in vitro and in vivo imaging, Sci Technol Adv Mat. 20 (2019) 337-355. doi:10.1080/14686996.2019.1590731.

[17] M. Fang, J. Li, J.A. Rudd, S.M. Wai, J.C.C. Yew, D.T. Yew, fMRI Mapping of cortical centers following visual stimulation in postnatal pigs of different ages, Life Sci. 78 (2006) 1197-1201. doi:10.1016/j.lfs.2005.06.030.

[18] P. Sauleau, E. Lapouble, D. Val-Laillet, C.-H. Malbert, The pig model in brain imaging and neurosurgery, Animal. 3 (2009) 1138-1151. doi:10.1017/s1751731109004649.

[19] M.M. Swindle, A. Makin, A.J. Herron, F.J. Clubb, K.S. Frazier, Swine as Models in Biomedical Research and Toxicology Testing, Vet Pathol. 49 (2011) 344-356. doi:10.1177/0300985811402846.

[20] E.M.A. Anas, H.K. Zhang, J. Kang, E. Boctor, Enabling fast and high quality LED photoacoustic imaging: a recurrent neural networks based approach, Biomed Opt Express. 9 (2018) 3852. doi:10.1364/boe.9.003852.

[21] J. Kang, C. Yoon, J. Lee, S.-B. Kye, Y. Lee, J.H. Chang, G.-D. Kim, Y. Yoo, T.-K. Song, A Systemon-Chip Solution for Point-of-Care Ultrasound Imaging Systems: Architecture and ASIC Implementation, IEEE T Biomed Circ S. 10 (2016) 412-423. doi:10.1109/tbcas.2015.2431272.

[22] G.-D. Kim, C. Yoon, S.-B. Kye, Y. Lee, J. Kang, Y. Yoo, T.-K. Song, A single FPGA-based portable ultrasound imaging system for point-of-care applications., IEEE T Ultrason Ferroelectr Freq Control. 59 (2012) 1386 1394. doi:10.1109/tuffc.2012.2339. 


\section{Tables}

Table 1. Expected and measured signal-to-noise ratio (SNR) at SSS in vivo for different frame averaging schemes. The asterisk marks follow the earliest point with statistical significance between $\bar{\Phi}_{S S S}$ and $\Phi_{L E D}$.

\begin{tabular}{|c|c|c|c|c|c|c|c|c|c|c|c|c|}
\hline & $N$ & 1 & 2 & 5 & 10 & 20 & 50 & 100 & 200 & 500 & 1,000 & 2,000 \\
\hline \multirow[t]{2}{*}{$690 \mathrm{~nm}$} & SNR $_{\text {est }}$ & -5.62 & -4.12 & -2.13 & -0.63 & $0.87^{*}$ & 2.86 & 4.37 & 5.88 & 7.86 & 9.42 & 10.96 \\
\hline & SNR $_{\text {LED }}$ & $\begin{array}{ll}-6.17 \pm \\
0.34\end{array}$ & $\begin{array}{|ll|}-4.40 \pm \\
0.31\end{array}$ & $\begin{array}{|ll|}-2.30 & \pm \\
0.53 & \\
\end{array}$ & $\begin{array}{|ll|}-0.75 & \pm \\
0.33 & \\
\end{array}$ & $\begin{array}{l}0.73^{ \pm} \\
0.26^{*}\end{array}$ & $\begin{array}{ll}2.72 & \pm \\
0.25 & \\
\end{array}$ & $\begin{array}{ll}4.27 & \pm \\
0.26 & \\
\end{array}$ & $\begin{array}{ll}5.68 & \pm \\
0.27 & \\
\end{array}$ & \begin{tabular}{|ll}
7.68 & \pm \\
0.29 & \\
\end{tabular} & \begin{tabular}{|ll}
9.19 & \pm \\
0.29 & \\
\end{tabular} & \begin{tabular}{|l}
$10.71 \pm$ \\
0.27
\end{tabular} \\
\hline \multirow{2}{*}{$850 \mathrm{~nm}$} & SNR $_{\text {est }}$ & -1.03 & 0.46 & $2.45^{*}$ & 3.96 & 5.46 & 7.46 & 8.97 & 10.46 & 12.44 & 13.96 & 15.47 \\
\hline & SNR $_{\text {LED }}$ & $\begin{array}{ll}-0.21 \pm \\
0.58 & \\
\end{array}$ & $\begin{array}{|ll|}1.30 & \pm \\
0.43 & \\
\end{array}$ & $\begin{array}{|ll|}3.50 & \pm \\
0.35^{*} & \\
\end{array}$ & $\begin{array}{|ll|}5.06 & \pm \\
0.34 & \\
\end{array}$ & $\begin{array}{|ll|}6.61 & \pm \\
0.33 & \\
\end{array}$ & $\begin{array}{ll}8.55 & \pm \\
0.28 & \\
\end{array}$ & $\begin{array}{l}10.07 \pm \\
0.29\end{array}$ & $\begin{array}{l}11.58 \pm \\
0.30\end{array}$ & \begin{tabular}{|l}
$13.57 \pm$ \\
0.29 \\
\end{tabular} & \begin{tabular}{|l}
$15.08 \pm$ \\
0.30 \\
\end{tabular} & \begin{tabular}{|l}
$16.58 \pm$ \\
0.30 \\
\end{tabular} \\
\hline
\end{tabular}

\section{Figures}

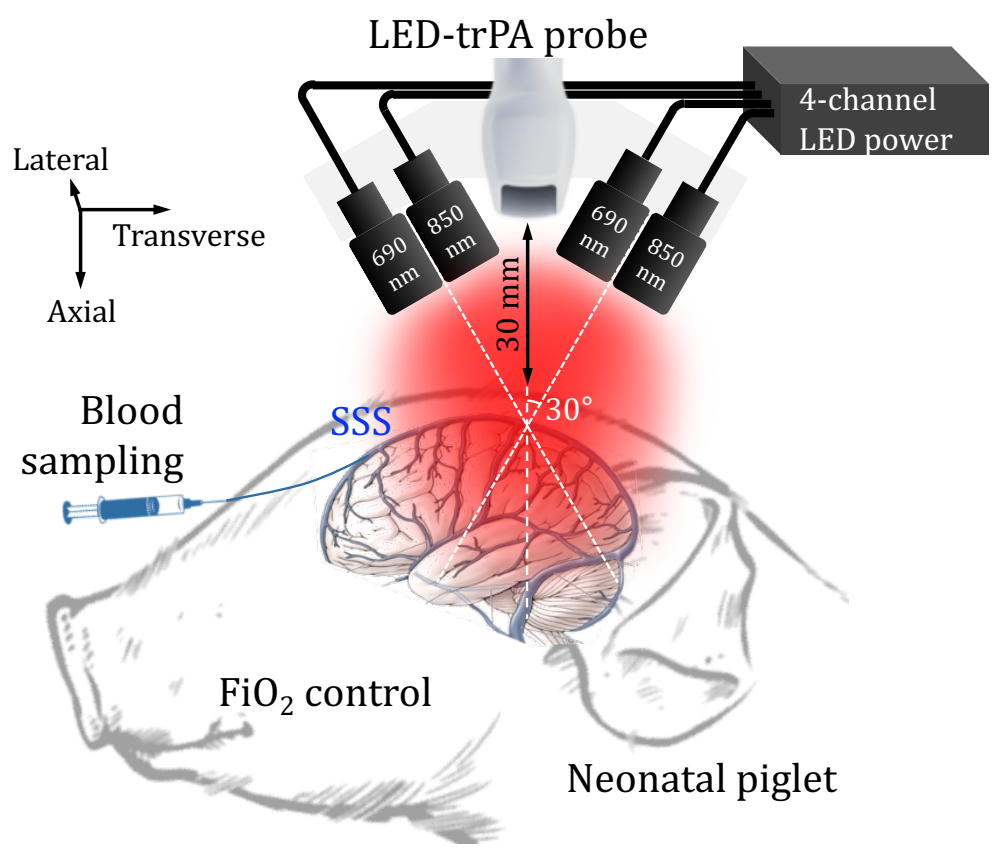

Fig. 1. Pulsed LED-based transcranial photoacoustic sensing (LED-trPA) system. Abbreviations: superior sagittal sinus, $\mathrm{SSS}$; fraction of inspired $\mathrm{O}_{2}, \mathrm{FiO}_{2}$. 
(a)

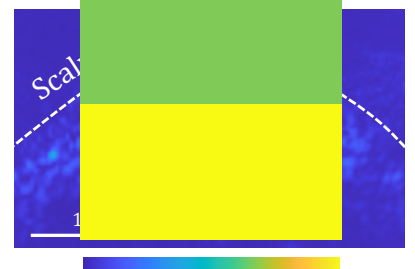

PA intensity (b)

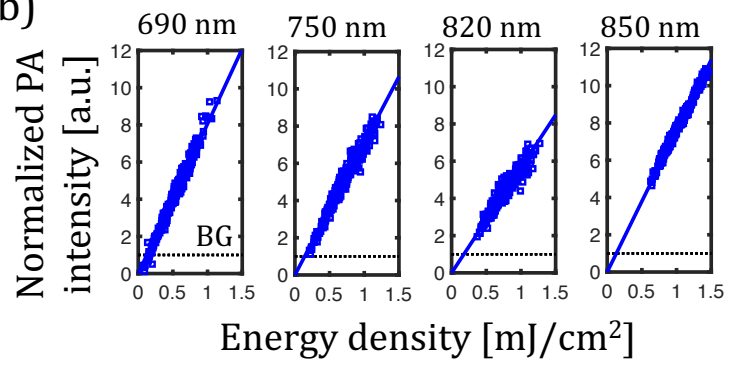

(c)

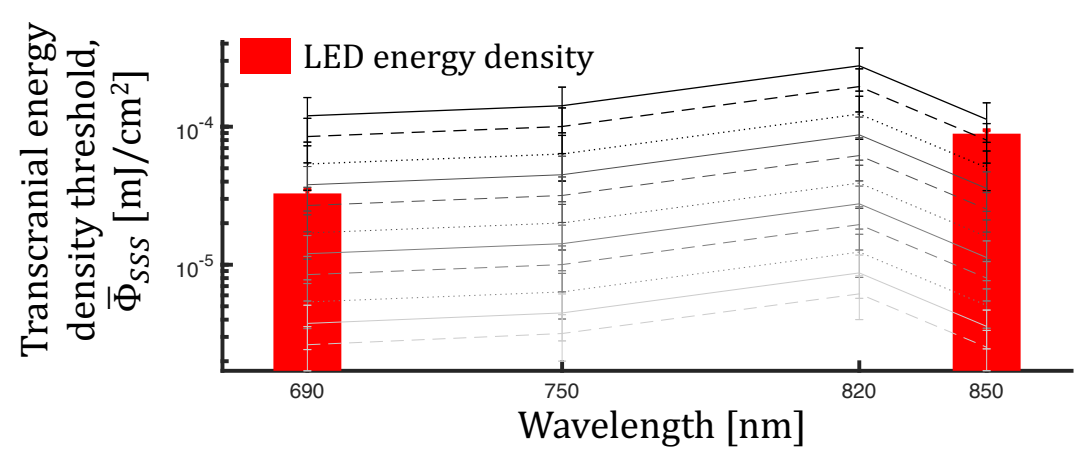

Fig. 2. Transcranial energy density threshold for SSS sensing, $\bar{\Phi}_{S S S}$. (a) exemplary PA cross-section of piglet head and region-of-interest at SSS. (b) Linear regression curves between energy density and PA intensity at SSS. (c) $\bar{\Phi}_{S S S}$ curves defining 0-dB SNR at SSS for 1, 2, 5, 10, 20, 50, 100, 200, 500, 1,000, and 2,000 frame averaging schemes (black to gray). Red bars indicate LED energy densities at 690 and 850 $\mathrm{nm}$. Abbreviations: superior cortical veins, SCV.

(a) Number of averaging frames

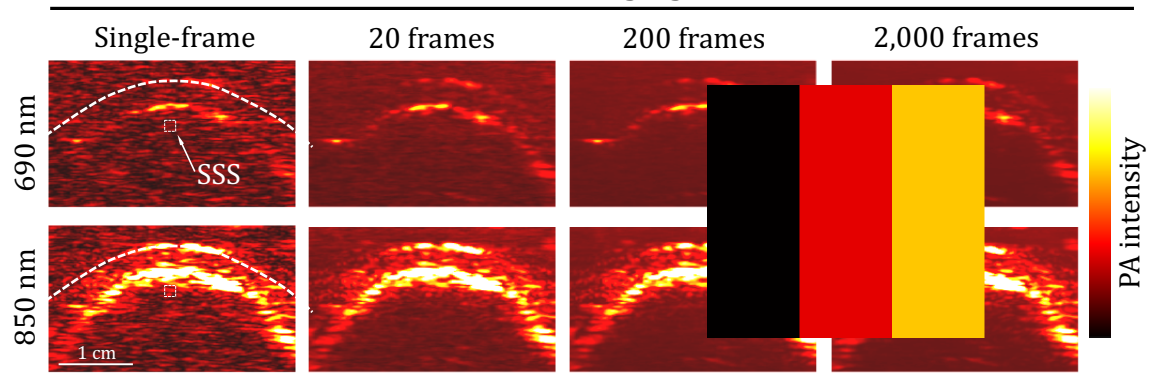

(b)

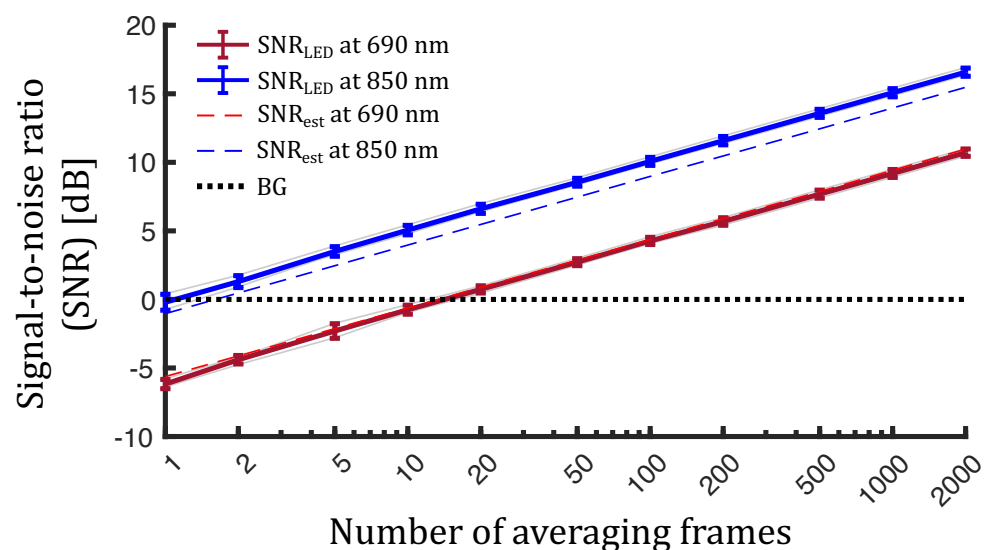

Fig. 3. (a) LED-trPA images at $690 \mathrm{~nm}$ and $850 \mathrm{~nm}$, processed with 1, 20, 200, 2,000 frame averaging schemes. (b) Comparison between SNR est and $\mathrm{SNR}_{\mathrm{LED}}$ at $690 \mathrm{~nm}$ and $850 \mathrm{~nm}$. 
(a) Number of averaging frames
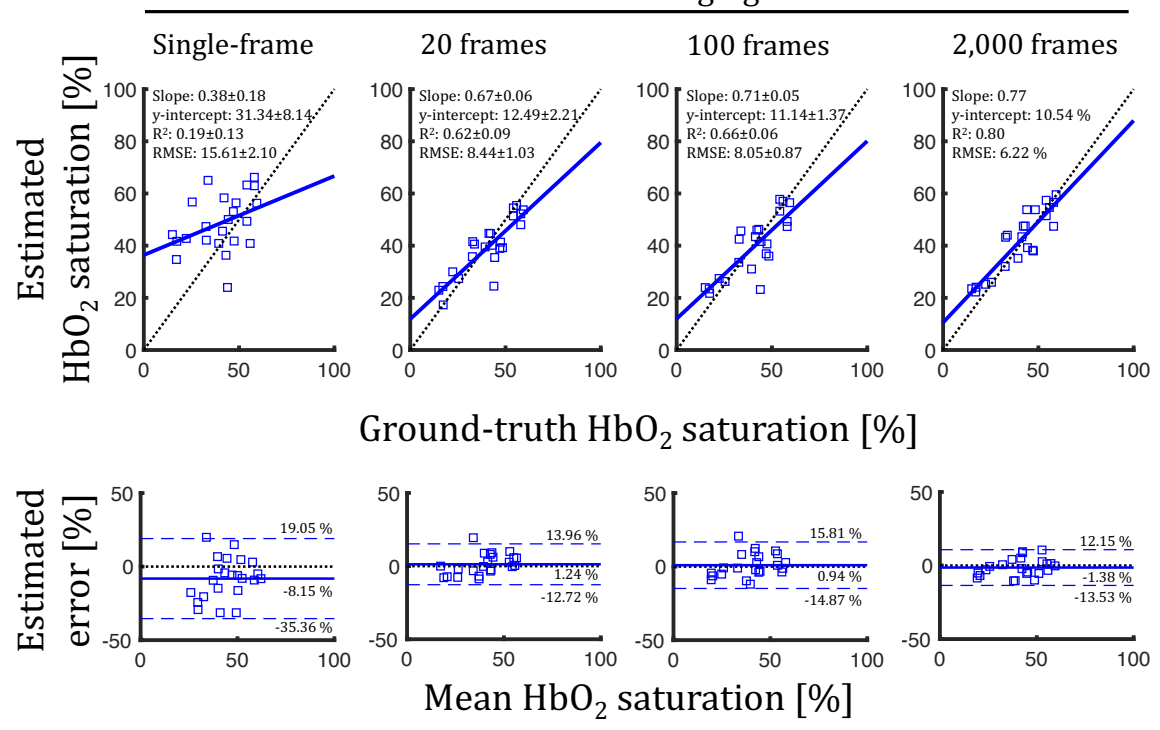

(b)
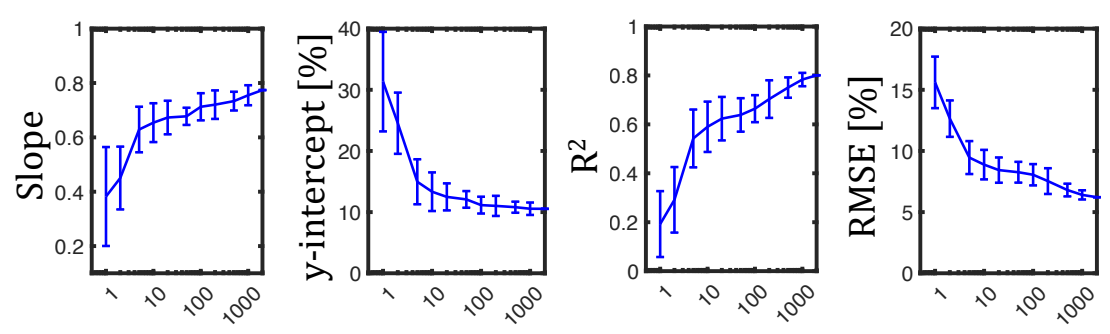

Number of averaging frames

Fig. 4. Correlation of estimated and ground-truth $\mathrm{HbO}_{2}$ saturation at SSS in neonatal piglets. (a) Linear regression curves and Bland-Altman estimation analysis for different frame averaging schemes. (b) Slope, $\mathrm{y}$-intercept, $\mathrm{R}^{2}$ and RMSE of the linear regression curves. 